\title{
Toward a large telescope facility for submm/FIR astronomy at Dome C
}

\author{
Gilles Alphonse Durand $^{\mathrm{a}^{*}}$, Vincent Minier ${ }^{\mathrm{a}^{* *}}$, Pierre-Olivier Lagage $^{\mathrm{a}}$, Emanuele Daddi $^{\mathrm{a}}$, Samir El \\ Khouloudi $^{\mathrm{a}}$, Nicola Schneider-Bontemps ${ }^{\mathrm{a}}$, Michel Talvard ${ }^{\mathrm{a}}$, Christian Veyssière ${ }^{\mathrm{b}}$, Gilles André \\ Durand $^{\mathrm{b}}$, Christian Walter ${ }^{\mathrm{b}}$, Lucia Sabbatinic ${ }^{\mathrm{c}}$, Zalpha Challita ${ }^{\mathrm{d}, \mathrm{g}}$, John W.V. Storey ${ }^{\mathrm{e}}$, Paolo Calisse ${ }^{\mathrm{f}}$, \\ Alain Pierre $^{\mathrm{g}}$, Maurizio Busso ${ }^{\mathrm{h}}$ \\ ${ }^{\mathrm{a} C E A}$, DSM, IRFU, Service d'Astrophysique, 91191 Gif-sur-Yvette, France; ${ }^{\mathrm{b}} \mathrm{CEA}$, DSM, IRFU, \\ SIS, France; ${ }^{c}$ University of Rome "La Sapienza", Department of Physics, Italy; ${ }^{\mathrm{d}}$ Laboratoire H.

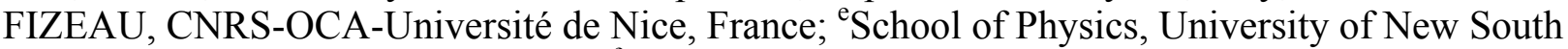

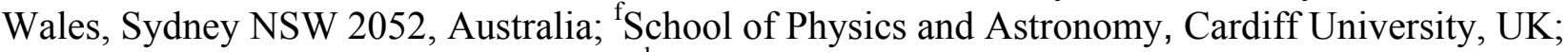 \\ gIPEV, 29280 Plouzané, France, ${ }^{\mathrm{h}}$ Dipartimeto di Fisica, Universita di Perugia, Italy
}

\begin{abstract}
Submillimetre astronomy is the prime technique to unveil the birth and early evolution of stars and galaxies in the local and distant Universe. Preliminary meteorological studies and atmospheric transmission models tend to demonstrate that Dome C might offer atmosphere conditions that open the $200-\mu \mathrm{m}$ atmospheric windows, and could potentially be a site for a large ground-based telescope facility. However, Antarctic climate conditions might also severely impact and deform any telescope mirror and hardware. We present prerequisite conditions and their associate experiments for defining a large telescope facility for submillimetre astronomy at Dome C: (1) Whether the submm/ THz atmospheric windows open from $200 \mu \mathrm{m}$ during a large and stable fraction of time; (2) The knowledge of thermal gradient and (3) icing formation and their impact on a telescope mirror and hardware. This paper will present preliminary results on current experiments that measure icing, thermal gradient and sky opacity at Dome C. We finally discuss a possible roadmap toward the deployment of a large telescope facility at Dome C.
\end{abstract}

Keywords: submillimetre astronomy, Antarctica, Dome C, SEFIRAA, ARENA.

*durandgs@cea.fr; **vincent.minier@cea.fr; http://irfu.cea.fr/Sap/Antarctica; Part of this work is supported by the ARENA, a European Commission CA network.

\section{SUBMM/FIR ASTRONOMY CONTEXT AND ANTARCTICA}

\subsection{Submillimetre astronomy and science drivers}

Far-infrared/submillimetre (FIR/submm - 100 to $1000 \mu \mathrm{m}$ ) astronomy is the prime technique to study the 'cold Universe' and unveil the birth and early evolution of planets, stars and galaxies. It is a relatively new branch of astronomy at the frontier between IR and radio astronomy. FIR/submm continuum observations are particularly powerful to measure the luminosities, temperatures and masses of cold dust emitting objects because dust enshrouded star-forming regions emit the bulk of their energy between 60 and $500 \mu \mathrm{m}$. The submm/FIR range of the spectrum or THz regime is also rich in several lines that are the only means to study the kinematical structure of the interstellar medium (ISM) of galaxies. They allow to probe different physical regimes, i.e. regions of widely different densities and temperatures, depending on their excitation levels and critical abundances. Observations at these wavelengths with a large telescope will primarily lead to breakthroughs in the study of star formation at all scales and understand its cosmic history back to the early Universe as well as in the understanding of galaxy evolution. Asteroids, debris disks, planet formation, dust origin in evolved stars, interstellar dust and polarisation of dust in the Universe are also potential science drivers for FIR/submm astronomy. An overview of FIR/submm astronomical science at Dome C is presented in Minier et al. (2008). Among the major science cases, a large, groud-based telescope facility would particularly lead to scientific breakthrough in two fields of astrophysics.

The origin of the stellar initial function and star formation in Milky Way and the Magellanic cloud: The problem of the origin of the distribution of stellar masses at birth, or initial mass function (IMF), is one of the most important open 
issues in astrophysics today. It is not only central in local star formation research, but also crucial to understand whether the IMF is truly universal, including in starburst galaxies and at high redshift, or likely to depend on local physical conditions such as metallicity, pressure, or temperature. As high-mass protostars emit the bulk of their energy between 60 and $500 \mu \mathrm{m}$ (Minier et al. 2005), submm continuum mapping is a unique tool to address this problem. The gain in angular resolution with a large ground-based telescope and the possibility to observe from 200 to $450 \mu \mathrm{m}$ at Dome C could also foresee observations of star formation beyond the Milky Way. In the Magellanic Clouds (LMC and SMC), the angular resolution of a $12-\mathrm{m}$ telescope at $200 \mu \mathrm{m}$ would allow observation with a spatial resolution of $1 \mathrm{pc}$. Dome $\mathrm{C}$ has also a privileged location to observe the Magellanic Clouds $\left(\mathrm{RA}=05 \mathrm{~h} \mathrm{Dec}=-69^{\circ}\right)$ and study the star formation rate in their gas-rich and metal-poor environment.

Cosmic history of star formation and galaxy evolution: The Cosmic Infrared Background (CIB) was discovered with the COBE satellite a decade ago. It results from the emission in the infrared (IR) of distant galaxies, the so-called infrared galaxies. These galaxies appear to emit more intensively in the IR than the most nearby galaxies. Two physical mechanisms may explain the high level of IR emission that is measured in the CIB. First the distant galaxies may form stars in a much more productive ways, through starburst mechanisms. The UV lights of high mass stars heat up the dust grains in the interstellar medium that re-emit in the IR and submm. Consequently, the Spectral Energy Distribution (SED) of these IR and submm galaxies peaks in the submm/FIR due to star formation. A second mechanism may be the effect of supermassive black holes in the active galactic nuclei (AGN) that emit in X-ray after swelling hot gas from nearby disrupted stars. Dust again absorbs the X-ray radiation and re-emit in the IR. The IR excess due to AGN manifests at $24 \mu \mathrm{m}$ mainly (Daddi et al. 2007). In both cases, the CIB provides astronomers with information on the galaxy formation and evolution, as well as on the history of cosmic star formation in the Universe. The CIB in the submm/FIR is still largely unexplored territory as compared to radio and optical/IR sky where all-sky maps of the background exist. Only small areas $\left(\sim 1 \mathrm{deg}^{2}\right)$ have been mapped up to date. Observations with space telescopes (Akari, Spitzer, and soon Herschel) should modify the current knowledge of the CIB and high-angular resolution observations of the new fields will be needed. Two major tasks could be accomplished in FIR/submm in order to progress in the understanding of the CIB and galaxy formation and evolution: To cense star formation in the distant Universe, to account for most of stellar mass in galaxies; To cense obscured (by dust) AGN activity by determining the bolometric luminosity of the associated IR galaxy. Major questions are: How massive ellipticals were formed, through merging ? What is the stellar initial mass function (IMF) for galaxy formation at high-z ? At what epoch(s) did most of the star formation takes place? What is the link between AGN and galaxy activity ? Observing in the submm/FIR at Dome C is an exciting opportunity if a telescope facility could resolve galaxies at $1<\mathrm{z}<3$ with a mass down to $10^{10} \mathrm{M}_{\odot}$. It will allow a proper estimate of their bolometric luminosity. This implies to go down to mJy sensitivities at submm/FIR wavelengths.

\subsection{Submillimetre astronomy context}

Two major submm facilities will become available in the coming years: the Herschel Space Observatory, a FIR/submm $(60-500 \mu \mathrm{m})$ telescope in Space, and ALMA, a ground-based mm-wave $(350 \mu \mathrm{m}-7 \mathrm{~mm})$ interferometer on the Chajnantor plateau in the northern Atacama desert. Both facilities will have their specific niches. Herschel will have the ability to carry out large area imaging surveys of both the distant Universe and the nearby interstellar medium in our own Galaxy. ALMA will make possible ultra deep searches for primordial galaxies, as well as detailed kinematical investigations of individual protostars. However, both Herschel and ALMA will have their own limitations. The Herschel telescope $(3.5 \mathrm{~m})$ will suffer from its only moderate angular resolution, implying a fairly high extragalactic confusion limit and preventing the study of individual protostars in all but the nearest star-forming clusters of our Galaxy. ALMA will suffer from a small field of view $\left(\sim 10^{\prime \prime}\right)$ and limited observable conditions in the FIR/submm, making extensive wide-field mapping impossible given the amount of time necessary to cover largestar-forming complexes and fields of primordial galaxies. Beside these two major facilities, there is a constellation of submm/FIR telescope projects in operation or in study, aboard balloons (e.g. BLAST, OLIMPO, PILOTE etc), aboard an airplane (SOFIA), aboard satellites (e.g. Akari; SPICA) and on high-altitude plateaux and mountains (e.g. APEX; CCAT, a 25-m telescope project). For instance, the APEX telescope at Chajnantor could allow $450-\mu \mathrm{m}$ observations as recently demonstrated in 2007 by the CEA ArTéMiS project (see Talvard et al., SPIE conference 7020).

Why evaluating the feasibility of installing a FIR/submm telescope at Dome C ? Beside Herschel and ALMA, there is thus a clear need for a large (>10 m) single-dish telescope (1) operating at 200-450 $\mu \mathrm{m}$ and providing (2) better angular resolution than Herschel and (3) wider-field mapping capabilities than ALMA, making large-scale mapping with a relatively good angular resolution (1") possible and well matched with thermal infrared space telescope (e.g. Spitzer). New sites are therefore intensively tested in the Andes (e.g. northern Chile) and in Antactica (e.g. Dome C, Dome A) to 
measure the atmospheric transmission in the 200-350-450- $\mu \mathrm{m}$ atmospheric windows. The stability of the atmosphere is an equally important parameter when comparing the sites and Dome $\mathrm{C}$ may stand out as being far more stable than Chilean sites (Minier et al. 2007 and reference therein). Equipped with FIR/submm imagers and spectrometers, a telescope at Dome C in Antarctica might be able to operate in all atmospheric windows between $200 \mu \mathrm{m}$ and $1 \mathrm{~mm}$, and very regularly at $450 \mu \mathrm{m}$ in wintertime. As a demonstration Antarctica is indeed a very good site for submm $/ \mathrm{mm}$ astronomy, US astronomers have built a submm telescope of $10 \mathrm{~m}$ (the SPT) at the South Pole (Ruhl et al. 2004). On a higher and more stable site, Dome C could become the European observatory in Antarctica.

\subsection{The concordia station: a potential submm observatory}

Dome $\mathrm{C}$ is the location of the French-Italian Concordia station that is connected to Dumont-D'Urville on the coast by either light planes or ground motorised raids for transporting heavy material. The Station building can now host about 15 people during winter and therefore allows experiments all year long (http://www.concordiastation.org/). Site testing and qualification of the site for optical and near-infrared astronomy have been conducted for many years by French, Italian and Australian teams. However, little efforts have been produced so far to evaluate the quality of the atmosphere, the climate constraints and the specificity of Dome C for a potential submm/FIR telescope. Calisse et al. (2004) undertook the measurement of the atmosphere opacity at $350 \mu \mathrm{m}$ during a summer period and found it comparable to that at South Pole. No direct assessment of the wintertime atmosphere transmission has ever been performed.

A major obstacle to carry out submm observations from ground is the water vapour in the atmosphere. Astronomical observations in the FIR/submm spectral bands (e.g. 200, 350, $450 \mu \mathrm{m}$ ) can only be achieved from cold, dry and stable sites with ground-based telescopes or from space to overcome the atmosphere opacity and instability that are mainly due to water vapour absorption and fluctuations in the low atmosphere. Chile currently offers the driest accessible (all-year long) sites on Earth, where the precipitable water vapour (PWV) content is often less than $1 \mathrm{~mm}$. The Chajnantor plateau $(5100 \mathrm{~m})$ hosts the ESO facilities for submm astronomy: the APEX 12-m telescope and the coming ALMA interferometer. However, FIR/submm observations at 200, 350, $450 \mu \mathrm{m}$ are only possible when PWV drops below 0.5 $\mathrm{mm}$, which occurs less than $30 \%$ of the observing time at the ALMA site and rarely over a continuous day. In addition, observing conditions in Chilean sites can progressively and frequently be degraded by climate phenomena like the Bolivian winter or El Niño that brings weather instabilities. Possibly global warming has a more severe impact on these sites than on Antarctica. Other potential Chilean sites at higher altitudes (e.g. Cerro Chajnantor, Sairecabur) presently undergo site testing (see Radford et al., this volume).

The plateau of Antarctica might possibly be a very privileged and alternative area for achieving astronomical observations in the submm/FIR range. The geographical and climate conditions are extreme, which favour low PWV in the atmosphere: the low sun cover, the isolation by the circumpolar stream of the Antarctic ocean and the high power of reflection of ice make Antarctica the coldest continent on Earth. It is also important to emphasize that snow precipitation is very low on the Antarctic plateau. The low pressure fronts do not penetrate into the inner plateau and remain located on the coast lines. In fact the inner part of the continent is a true desert: on an area of 5 millions of $\mathrm{km}^{2}$, snow precipitation is about $5 \mathrm{~cm}$, and often less than $2 \mathrm{~cm}$ on the highest Domes. As a consequence, the PWV at Dome $\mathrm{C}$ is very low and expected lower than at Chajnantor in average. However, a proper comparison between Dome C and other sites should be based on the comparison of the transmission, the linewidth of the atmospheric windows and their stability, and the level of skynoise. The transmission and the linewidth of the windows mainly depend on the PWV, temperature and pressure, while the sky noise depends on the water vapour cell fluctuations in the low atmosphere (see Minier et al. 2008).

Preliminary site testing in summertime and modelling predict that the French-Italian Concordia base at Dome $\mathrm{C}$ in Antarctica is a potentially remarkable site on Earth for FIR/submm astronomy (see Minier et al. 2008 and references therein for a review). The atmospheric transmission and stability are probably always ideal for observations at 350 and $450 \mu \mathrm{m}$. For regular ( $>100$ days) use of telescope time for the $350-450 \mu \mathrm{m}$ windows and complementary observations at $200 \mu \mathrm{m}$, Dome C might become a very attractive site. However, a complete assessment of the $200-\mu \mathrm{m}$ opacity of the atmosphere is now crucial for concluding whether Dome $\mathrm{C}$ is better than any other known sites in the Chilean Andes and more generally on Earth. This will be achieved by SUMMIT in 2008 and 2009. Skynoise will also be measured by the CAMISTIC bolometer camera in 2010. Another crucial assessment is the understanding of the effects of temperature gradients in the inversion temperature layer between 0 and $30 \mathrm{~m}$ and icing formation during polar nights on telescope hardware and operation. Nighttime cooling of ice sheet by radiation to space creates a powerful temperature gradient and generates near surface level inversion winds where air, cooled by contact with the surface, flows down the gently sloped 
interior of the plateau (Swain \& Gallée 2006). The studies of these constraints are realised under the GIVRE experiments, which are currently in operation at Dome C.
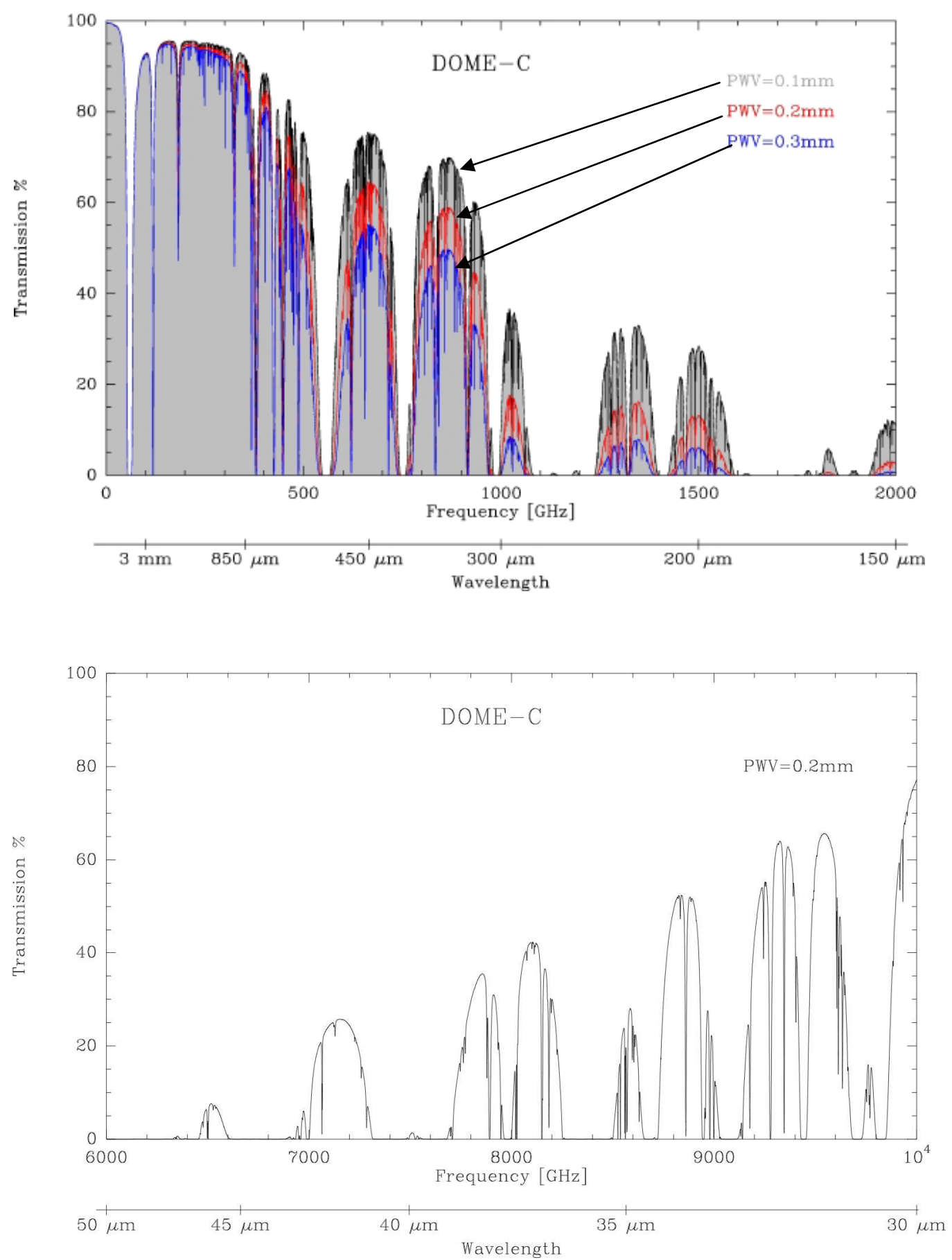

Figure 1: Modelled transmission for Dome $\mathrm{C}$ with the MOLIERE code. Transmissions were estimated using PWV=0.1, 0.2 and $0.5 \mathrm{~mm}$ in the submm/mm range $(150 \mu \mathrm{m}$ to $3 \mathrm{~mm}$; upper figure) and $\mathrm{PWV}=0.2 \mathrm{~mm}$ the far-infrared range (lower figure). Many observing windows open between 30 and $50 \mu \mathrm{m}$. The $200 \mu \mathrm{m}(1500 \mathrm{GHz})$ windows opens at Dome C under PWV $<0.5 \mathrm{~mm}$. Note: MOLIERE (Microwave Observation LIne Estimation and REtrieval) is a versatile forward and inversion model for the millimetre and submillimetre wavelengths range, used in many aeronomy and some astronomy applications (Urban 2004). 


\section{PREREQUISITE BEFORE INSTALLING A LARGE TELESCOPE FACILITY}

Is Dome $\mathrm{C}$ really the best site on Earth for the installation of a large submm-wave telescope ? How does the polar environment impact hardware? A site testing campaign in wintertime has specifically been designed for FIR/submm astronomy. Three prerequisites are now tested at Dome $\mathrm{C}$ before launching (or not) any advanced concept study: (1) whether the submm/THz atmospheric windows open from 200 micron during a large and stable fraction of time; (2) the knowledge of the thermal gradient and (3) icing formation and their impact on a telescope mirror and hardware. In parallel atmospheric modelling and meteorological data analysis will be carried out.

\subsection{Transmission at $200 \mu \mathrm{m}$ - The SUMMIT experiment}

A major obstacle to carry out submm observations below 500 microns from ground is the atmosphere as well as the harsh environment of the potential Earth site (high altitude deserts; Antarctica). Preliminary meteorological studies and atmospheric transmission models tend to demonstrate that Dome $\mathrm{C}$ might offer atmosphere conditions that open the 200$\mu \mathrm{m}$ windows. A submm tipper, SUMMIT in collaboration with UNSW/Sydney, has started operating in early 2008 for measuring sky opacity at 200 microns.

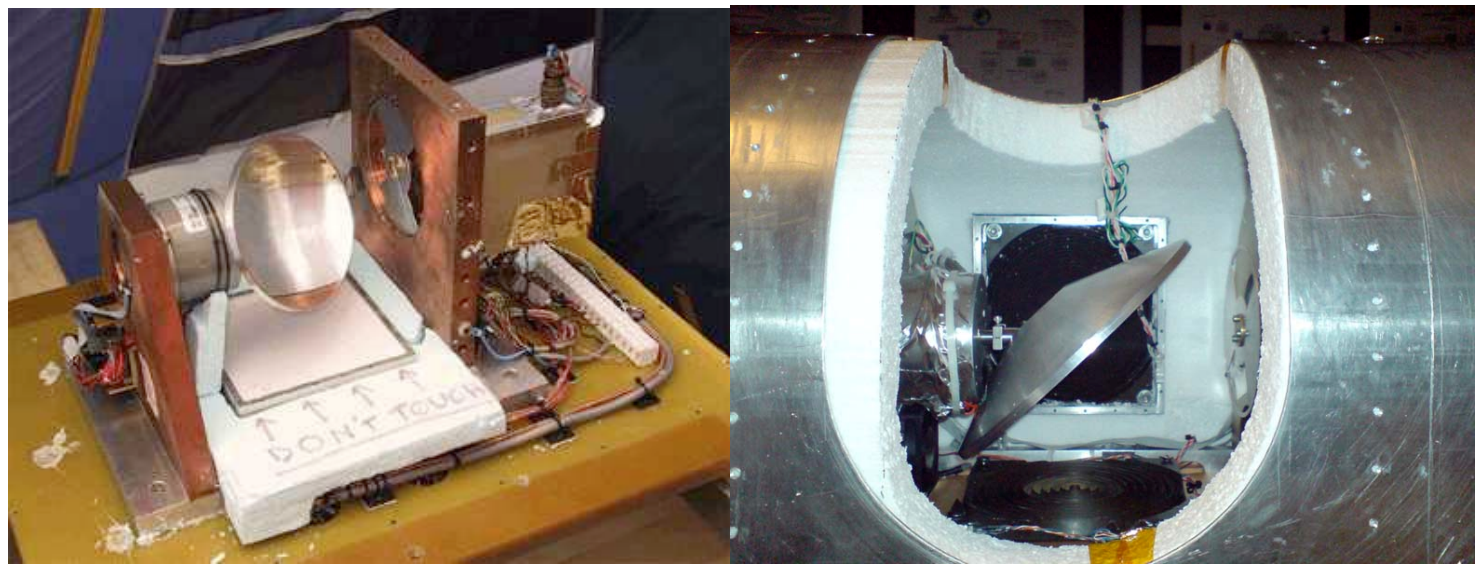

Figure 2: SUMMIT. Left: Inside with the mirror and the chopper wheel. Right: For 2008 campaign at Concordia the warm black body is kept warm in the airflow, heated by resistors. The reference black body at the rear is in contact with the cold aluminum cover.

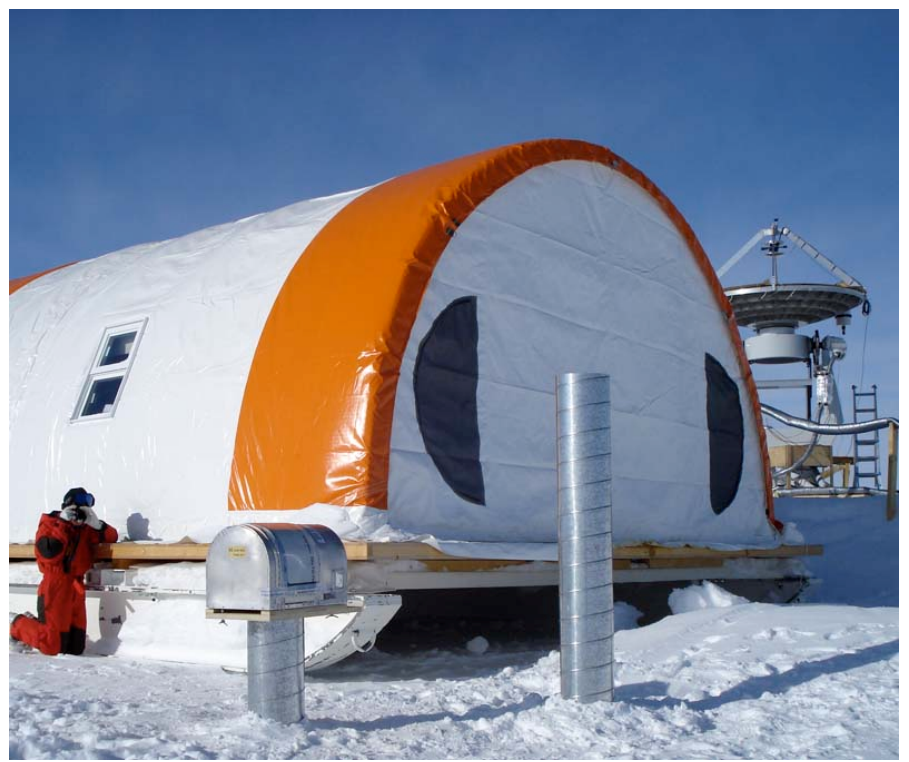

Figure 3: SUMMIT in operations at Concordia with Cochise at the rear. 


\subsection{Thermal gradient between 0 and $46 \mathrm{~m}$}

Common prerequisites for the design of unmanned telescopes at altitudes up to $46 \mathrm{~m}$ include the ongoing measurements of wind, humidity and temperature. Our team currently performs these measurements. The probes consist of 2 horizontal disks (CD like) distant by $10 \mathrm{~mm}$ to be used as sun shields. They are black inside, polished aluminium up and down. They are defrosted every 2 minutes for duration of 2 minutes. Inside is placed a thin aluminium cylinder (height $8 \mathrm{~mm}$, $ø 10 \mathrm{~mm})$ equipped with a Pt1000 small sensor and a small heater $(0.25 \mathrm{~W})$ working together with disks heaters. The temperature sensor is red every 10 seconds continuously. Turbulence and air temperature stability might be derived afterward.

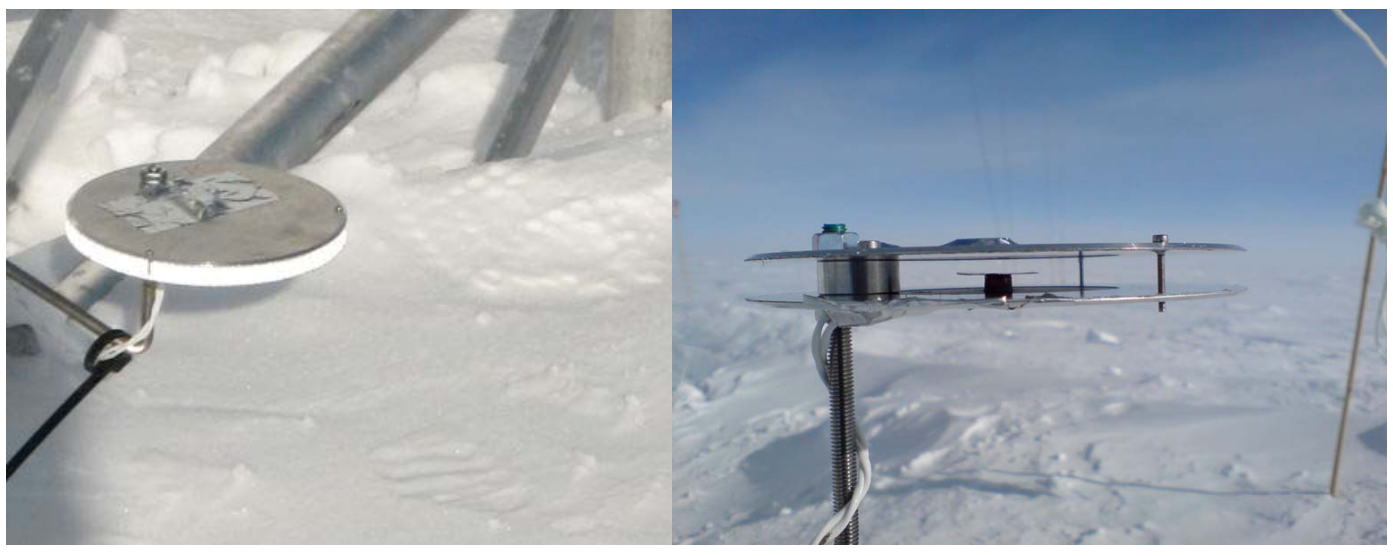

Figure 4: Left: Shielding of the thermal probes, the sunlight is back-reflected by the lower disk and forward reflected by the upper disk. Right : Pt1000 mounted on a ø20 mm disk protected by ø150mm disks.

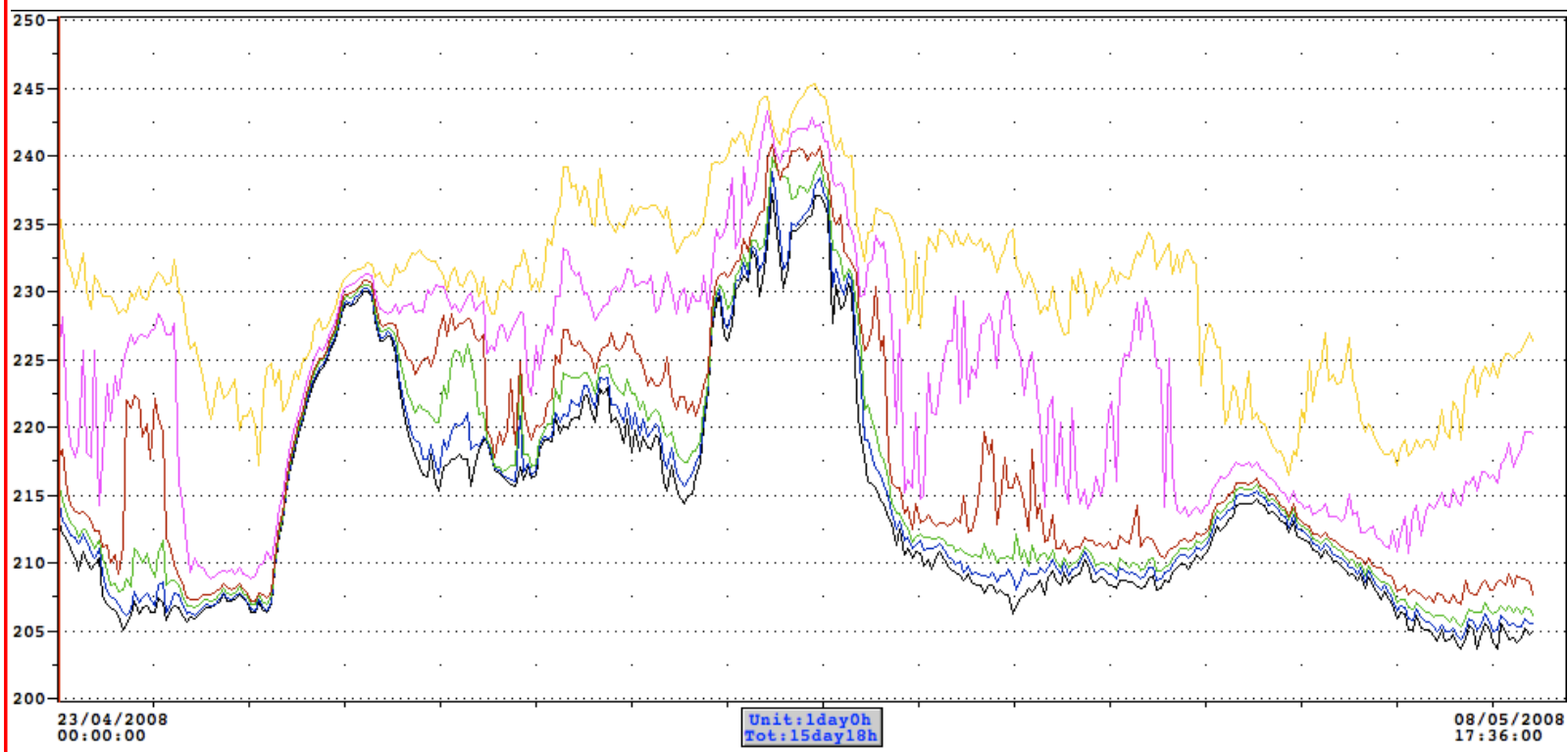

Figure 5: Temperatures in $\mathrm{K}$ measured at $0.2 \mathrm{~m}, 2,5,12,24$ and $46 \mathrm{~m}$. Note that temperatures are lower at $0.2 \mathrm{~m}$ than at 46 $\mathrm{m}$ high. Variations of temperature with height go up to $20 \mathrm{~K}$. 


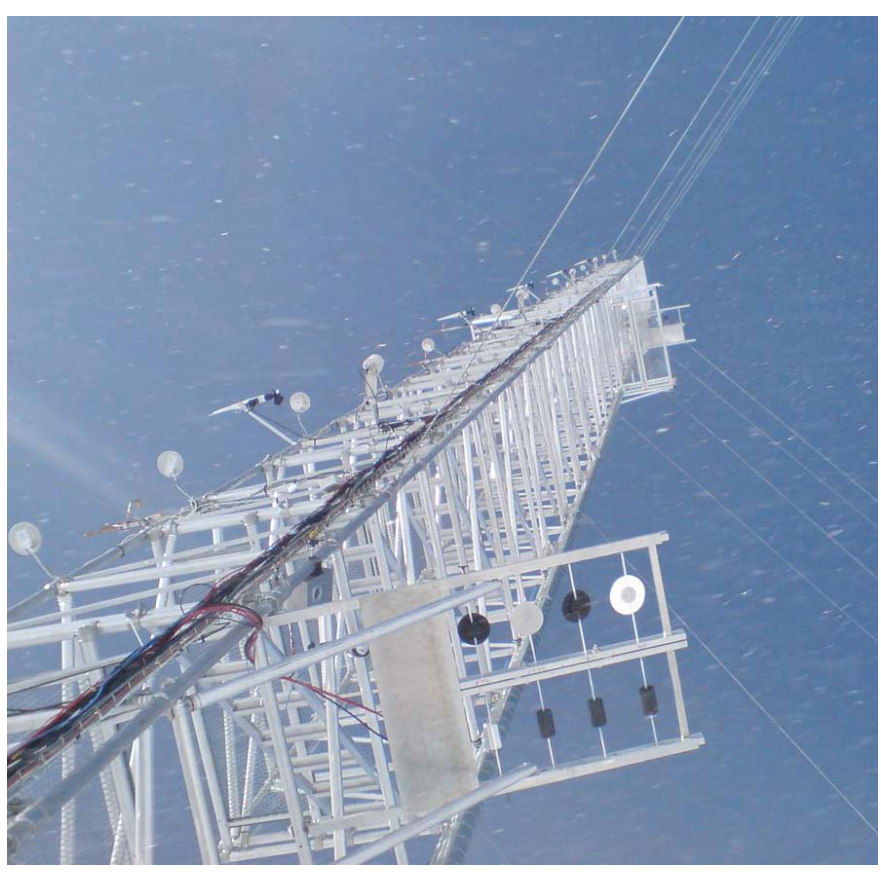

Figure 6: The temperature cells can be seen on the left of the tower. The frost cells are placed on two platform at $5 \mathrm{~m}$ and $30 \mathrm{~m}$ on the right of the $46 \mathrm{~m}$ mast.

\subsection{Icing formation on telescope and hardware}

Dome $\mathrm{C}$ climate conditions severely impact and deform any telescope mirror and hardware. Whatever the telescope at Dome $\mathrm{C}$ the combination of little wind and high heat lost by radiation during the polar winter, results into a large thermal inversion and strong icing on all hardware objects. Our team currently tests different ways of protecting the instruments against frost.

16 temperature and wind measurements are performed from 0 to $46 \mathrm{~m}$, with closer altitude resolution near the ground. On an aluminium blade, we shall blow a $0.4 \times 0.6 \mathrm{~m}^{2}$ glossy aluminium disk with fresh air blown from the ground in order to check if dry air may clean a surface in altitude (Figure 7).

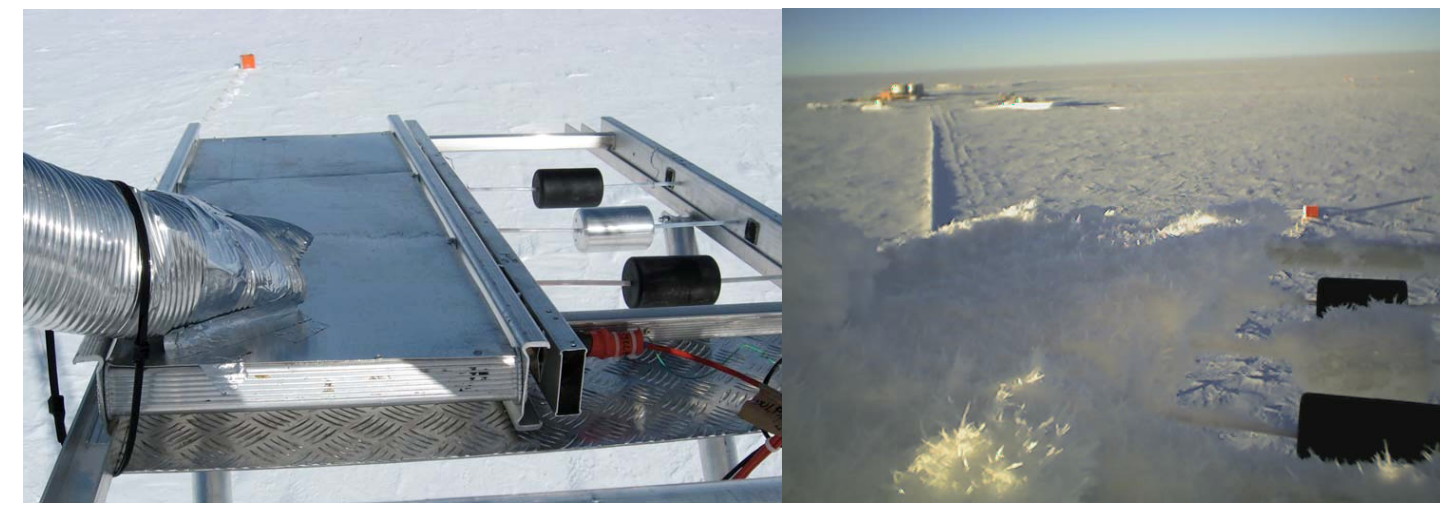

Figure 7: Left: At 30m a shiny aluminium surface is blowed with dry air from the ground level, near the 3 heated frost cells. Right: At $30 \mathrm{~m}$ up to $6^{\circ} \mathrm{C}$ above ambient are sometimes needed to keep the cells clear of frost. The blower is not active on this photo. The crystals are typical frost built-up. 


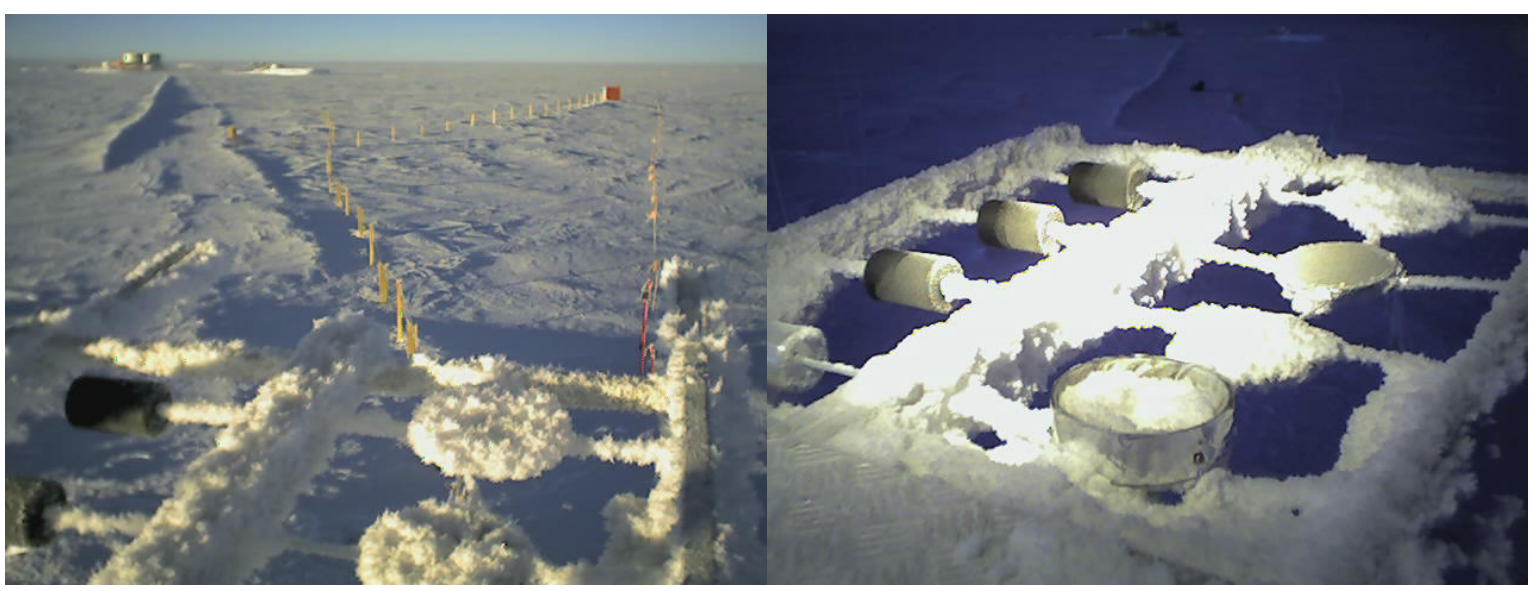

Figure 8: Left: On 17 april 2008, the frost has covered all the non-heated areas at $5 \mathrm{~m}$ altitude. A heater at $3^{\circ} \mathrm{C}$ above ambient is needed to protect from frost built-up. Right: At $5 \mathrm{~m}$ altitude a disk is surrounded by a heated ring to protect ice builtup on a simulated telescope. On this view the warming up of the ring $\mathrm{T}$ ambient $+3^{\circ} \mathrm{C}$ cannot stop the ice.

The COCHISE telescope (Roma 3 University) has been equipped by a system that will monitor the formation of frost on the telescope primary mirror and will try to stop its formation by heating for instance (Figure 9).

The measurements in process at Concordia will describe how much the air is super saturated at $5 \mathrm{~m}$ and $30 \mathrm{~m}$. The strong temperature gradient blocks the air mixture. During 2007 winter campaign, the frost probes at $7 \mathrm{~m}$-altitude indicated super saturation of $75 \%$ above $100 \%$.

This is highly important as frost forms very quickly at Dome $\mathrm{C}$ on any piece of hardware. In the perspective of unmanned telescopes during polar winter, automatic removal of frost will be necessary for all type of telescope.

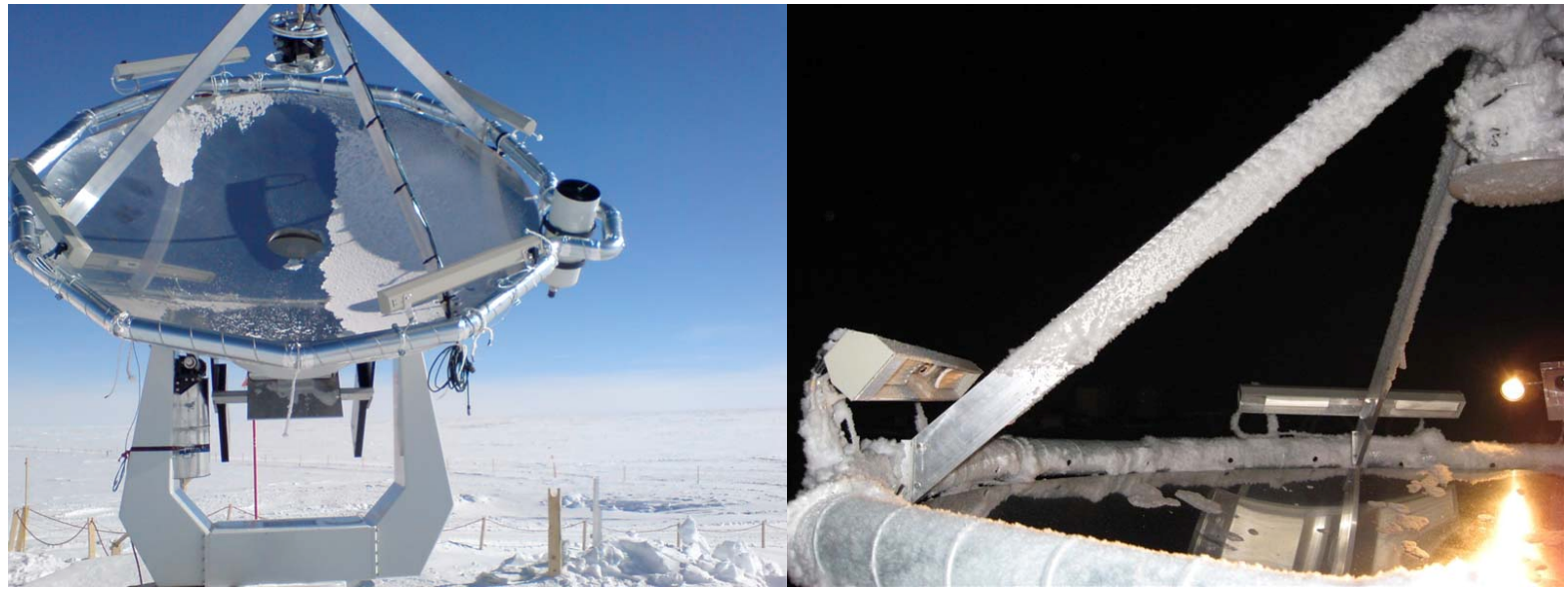

Figure 9: Left: The Italian 2.6m telescope Cochise equipped with infrared heaters, 24 holes to blow dry air and a heating system on the mirror. An efficient combination is a small warm up of the telescope to make the surface slippery in addition to blowers to flush the snow. Right: The infrared heaters remove the snow from the M2 support. This unprotected mirror accumulates frost toward the bottom. The M1 mirror in horizontal position is nearly clean. By tilting it, the snow would fall down. 


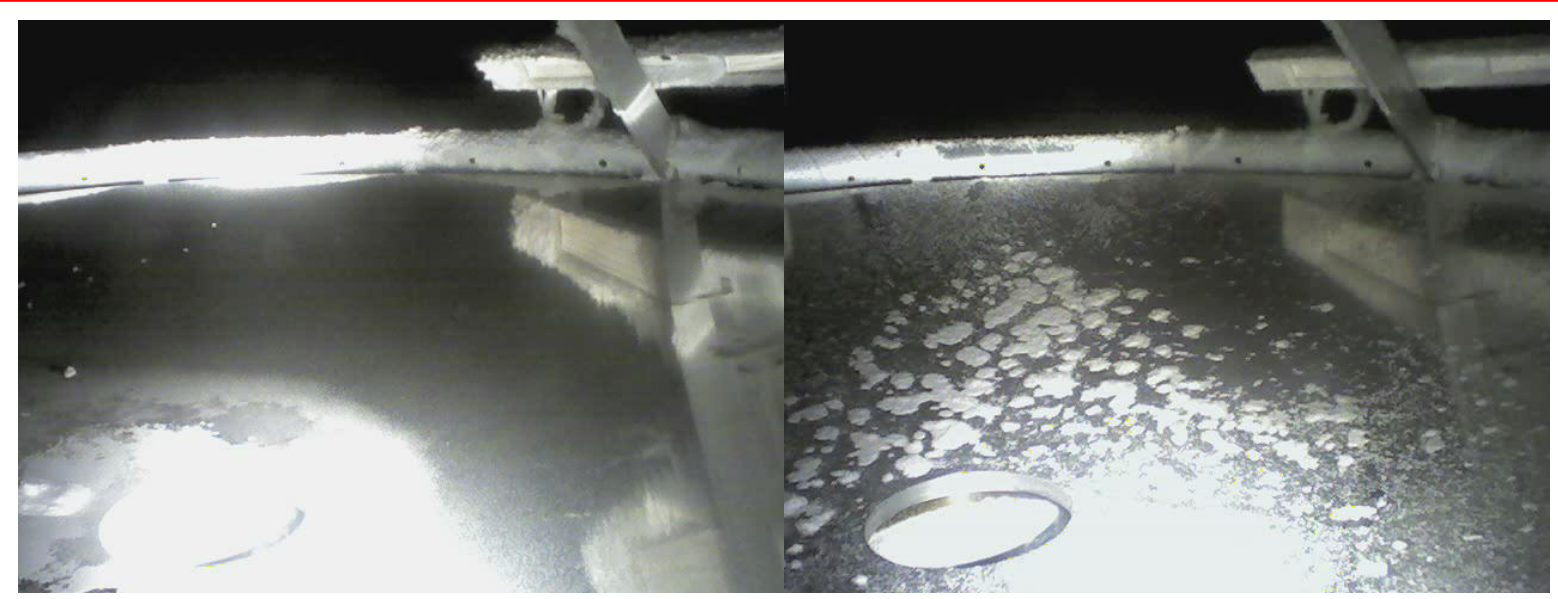

Figure 10: Left: On this web-cam photo, the mirror is heated $5^{\circ} \mathrm{C}$ above ambient. The snow is pushed toward the centre of the mirror by both gravity and the blower. Right: Without a cleaning anti-frost system, the mirror is rapidly covered with frost and snow.

\section{ROADMAP FOR SUBMM ASTRONOMY AT DOME C}

\subsection{Scientific and instrumental requirements}

Preliminary specifications on a potential telescope facility at Dome $\mathrm{C}$ are based on the science driver requirements that are presented in Minier et al. (2008). In all science cases, a relatively high angular resolution ( $\sim 1 ")$ is required to address key questions. This is the most important gain with respect to space telescopes that are still limited in diameter. Observations at $200-450 \mu \mathrm{m}$ are fundamental for constraining key physical parameters such as the luminosity and temperature from the spectral energy density of dust emission. Among the major science cases, the galaxy evolution and star formation cases require large areas $\left(1 \mathrm{deg}^{2}\right)$ to be mapped. While the ALMA interferometer can supply high angular resolution and will mainly study gas properties with spectral lines, ALMA cannot produce large area maps at 200-450 $\mu \mathrm{m}$ in a reasonable amount of time due to the limitation by the primary beam size ( $\sim 10 ")$ and atmosphere transmission. It cannot reach the THz $(1000-1500 \mathrm{GHz})$ spectral line regime either. A large aperture telescope $(\sim 25 \mathrm{~m})$ such as that proposed in the CCAT project for Cerro Chajnantor (Radford et al., this volume) would fulfil all the above requirements. A better site than Cerro Chajnantor is required to perform regular, ground-based observations at $200 \mu \mathrm{m}$. An alternative possibility to a large single dish-telescope (such as CCAT) would be an array of medium-size telescopes in a very compact baseline configuration that would simulate a 25-m dish sensitivity and angular resolution.

Dome C might offer the best alternative on Earth to reach the 200-350-450- $\mu \mathrm{m}$ windows. However, the extreme difficulty to work at Dome $\mathrm{C}$ argues for simple, robust, and unmanned experiments, especially after February when winter-over staff cannot maintain instruments outside, and the temperature rapidly drops to $-60^{\circ} \mathrm{C}$. Work on site must then be reduced to the installation and maintenance in the short summer period and operation in polar wintertime. Such constraints need to test technologies with prototype and pathfinder telescopes.

\subsection{CAMISTIC}

The CAMISTIC project aims to install a filled bolometer-array camera with $16 x 16$ pixels on a small telescope, possibly the IRAIT telescope (Tosti et al. 2006), at Dome C. CAMISTIC will explore the $200-\mu \mathrm{m}$ (i.e. THz) windows for groundbased observations. CAMISTIC will perform site testing on the atmospheric transmission and sky noise in the $200-\mu \mathrm{m}$ range. Sky noise measurements will be performed through sky imaging with the whole bolometer array. On a 80-cm telescope (IRAIT), the near field is located at $1.6 \mathrm{~km}$ for $200 \mu \mathrm{m}$. This means that the overlap between the bolometer pixel beams will be greater than $50 \%$ until about $1 \mathrm{~km}$, and above the near-field limit, the pixel beams completely diverge. If the water vapour cells responsible for the fluctuations in the atmosphere background power (i.e. sky noise) are located below about $1 \mathrm{~km}$, the pixels "see" through the same atmosphere column and sky noise. However, if these water vapour cells are above $1.6 \mathrm{~km}$, then non adjacent pixels on the array "see" through different atmosphere column and can potentially provide a map of the sky noise. 


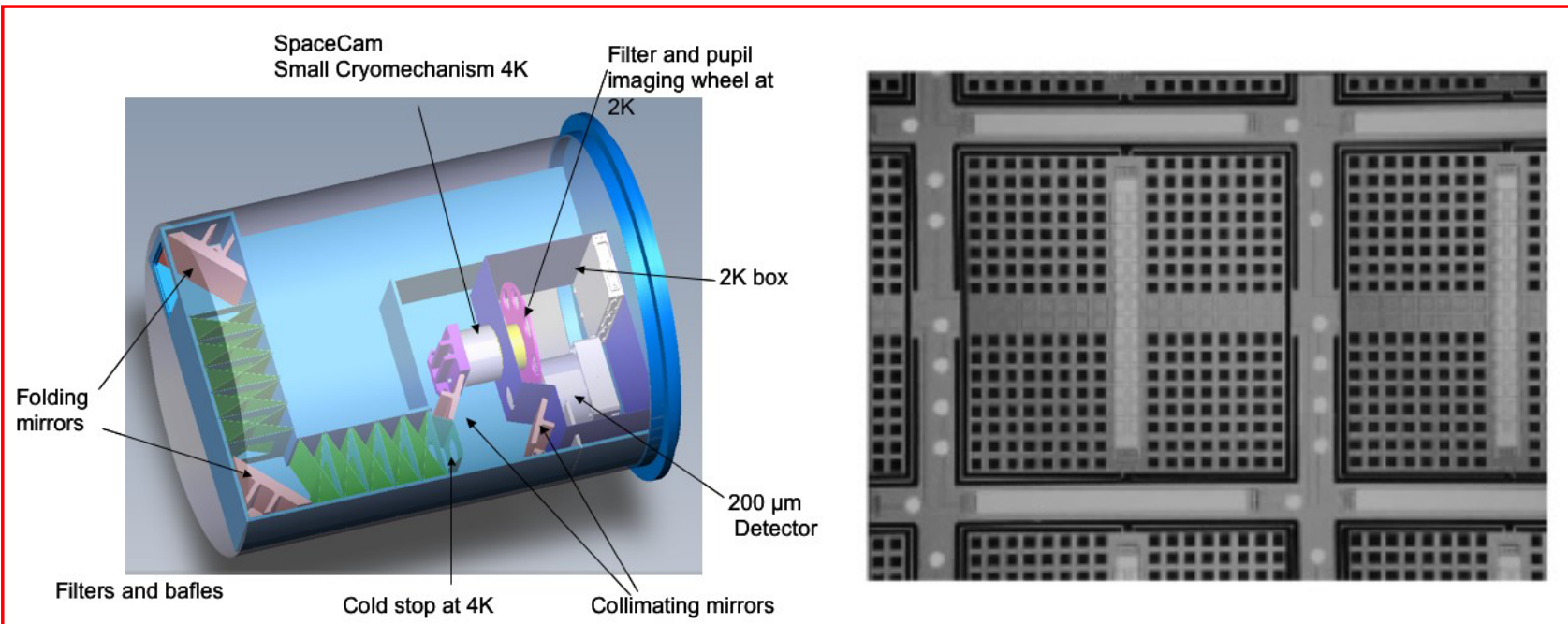

Figure 8: Left: View of the cryostat and optics to be installed on IRAIT in 2010. Right: View on a bolometer pixel of a 256pixel bolometer unit similar to the ArTéMiS and PACS bolometer units (Talvard et al, SPIE conference 7020).

\subsection{CAMISTIC on PILOT}

Preliminary studies have started to interface CAMISTIC with PILOT. PILOT is a $2.4 \mathrm{~m}$ wide-field optical/infrared telescope planned for Dome $C$ that could also host a submm imager (Burton et al. 2005). To take advantage of the unique site conditions, it will be on a tower above most of the turbulent boundary layer, it will be thermally controlled, it will have fast tip-tilt guiding, and it will have diffraction-limited optics across the 1 degree field (see Saunders et al., this volume).

\subsection{Toward a large telescope facility?}

There are different options for implementing a submm/FIR telescope facility at Dome C. The current approach exploits the available IR and mm telescopes (e.g. 80-cm IRAIT, 2.6-m COCHISE) on site to test the polar constraints and the quality of the atmosphere for astronomical observations. The goal of our experiments would be to have a final assessment of the site characterization for the submm wavelengths by 2010. This work will prepare future large projects by learning about polar telescope engineering, unmanned control, telescope foundations, wind and ice formation on mirrors, active surfaces at $\mathrm{T}<-70^{\circ} \mathrm{C}$, stability and transparency of submm/FIR windows, and optical designs. Alternative energy supply would also be needed such as photovoltaic panels (polar summer), 50-m high wind turbines and $\mathrm{H}_{2}$ fuel cells (polar winter) to power observatory facilities and the Concordia station. A more straightforward approach is the installation of a "winterized" 12-m ALMA-type antenna that could both undertake unique world-class science and pathfinder experiments. Finally, Dome $\mathrm{C}$ could also, if revealed as an amazing site for submm astronomy, hosts a demonstrator for a far-infrared interferometer. Such an interferometer with short baselines could be a direct detection interferometer with bolometers as imagers of the interferogram. It could demonstrate technology feasibility in the R\&D future Far-IR Interferometer space mission.

\section{REFERENCES}

[1] Calisse, P., Ashley, M.C.B., Burton, M.G. et al., PASA, 21, 256 (2004)

[2] Burton, M. G., Lawrence, J. S., Ashley, M. C. B. et al., PASA, 22, 199 (2005)

[3] Daddi E., Alexander, D.M., Dickinson, M. et al. ApJ, 670, 173 (2007)

[4] Minier, V., Burton, M.G., Hill, T. et al. A\&A, 429, 945 (2005)

[5] Minier, V., Durand, G., Lagage, P.-O., et al. ARENA-1 conference, EAS Publications Series, 25, 321 (2007)

[6] Minier, V., Olmi, L., Lagage, P.-O. et al. ARENA-2 conference, in press. (2008) Astro-ph: arXiv:0805.2487 http://irfu.cea.fr/Sap/Antarctica

[7] Ruhl, J., Ade, P.A.R., Carlstrom, J.E. Proceeding of SPIE, Millimeter and Submillimeter Detectors for Astronomy II. J. Zmuidzinas, W.S. Holland and S. Withington (eds), 5498, 11 (2004)

[8] Swain, M. \& Gallée, H., PASP, 118, 1190 (2006) 
Please verify that (1) all pages are present, (2) all figures are acceptable, (3) all fonts and special characters are correct, and (4) all text and figures fit within the margin lines shown on this review document. Return to your MySPIE ToDo list and approve or disapprove this submission.

[9] Tosti, G., Busso, M., Nucciarelli, G. et al. Ground-based and Airborne Telescopes. Stepp, Larry M.. Proceedings of the SPIE (eds), 6267, 62671 (2006)

[10] Urban, J., Baron, P., Lautié, N., Schneider, N., et al. J. Quant. Spectros. Radiat. Transfer 83, 529 (2004) 